\title{
Characterizing Model Completeness Among Mutually Algebraic Structures
}

\author{
Michael C. Laskowski
}

\begin{abstract}
We characterize when the elementary diagram of a mutually algebraic structure has a model complete theory, and give an explicit description of a set of existential formulas to which every formula is equivalent. This characterization yields a new, more constructive proof that the elementary diagram of any model of a strongly minimal, trivial theory is model complete.
\end{abstract}

\section{Introduction}

In [5], which borrows heavily from [4], it is shown that for any mutually algebraic structure $M$ (see Definition 1.4), its elementary diagram, which we denote by $T(M)$, has a near model complete theory. Indeed, Definition 1.6 describes a specific class $\mathcal{E}$ of existential $L(M)$-formulas, and every $L(M)$-formula is $T(M)$-equivalent to some Boolean combination of formulas from $\mathcal{E}$.

In earlier papers, it was shown that under stronger hypotheses on the theory of $M$, the elementary diagram $T(M)$ has a model complete theory. Indeed, in [3], Goncharov, Harizanov, Lempp, McCoy, and the author prove that the elementary diagram of every model of a strongly minimal, trivial theory is model complete. In [2], this result was strengthened by Dolich, Raichev, and the author to give the same result for any model of an $\aleph_{1}$-categorical, trivial theory of Morley rank 1 . In both instances, it follows that every $L(M)$-formula is equivalent to an existential formula, but the proofs do not give a specific description of a "minimal set" of existential formulas needed to describe all $L(M)$-formulas.

The main theorem of this short note, Theorem 2.4, characterizes when the elementary diagram of a mutually algebraic structure $M$ has a model complete theory (as opposed to simply being near model complete). Moreover, we display a set $\mathcal{P}$ of easily understood existential formulas, ${ }^{1}$ and show that $T(M)$ is model complete if and only if every $L(M)$-formula is $T(M)$-equivalent to an element of $\mathcal{P}$. Then,

Received May 31, 2012; accepted July 1, 2013

2010 Mathematics Subject Classification: Primary 03C10; Secondary 03C45

Keywords: model complete, mutually algebraic, strongly minimal, trivial

(C) 2015 by University of Notre Dame 10.1215/00294527-3132815 
in the third section, we indicate that these conditions hold for models of either of the two types of theories described above.

We conclude the Introduction by recalling the major definitions and results from [4] and [5].

Definition 1.1 When we write a tuple $\bar{z}$ of variable symbols, we assume that the elements of $\bar{z}$ are distinct, and $\operatorname{range}(\bar{z})$ denotes the underlying set of variable symbols. A proper partition $\bar{z}=\bar{x}^{\wedge} \bar{y}$ satisfies $\lg (\bar{x}), \lg (\bar{y}) \geq 1$, $\operatorname{range}(\bar{x}) \cup \operatorname{range}(\bar{y})=\operatorname{range}(\bar{z})$, and $\operatorname{range}(\bar{x}) \cap \operatorname{range}(\bar{y})=\emptyset$. We do not require $\bar{x}$ be an initial segment of $\bar{z}$, but to simplify notation, we write it as if it were.

Definition 1.2 Let $M$ denote any $L$-structure. An $L(M)$-formula $\varphi(\bar{z})$ is $m u$ tually algebraic if there is an integer $N$ so that $M \models \forall \bar{y} \exists \leq N \bar{x} \varphi(\bar{x}, \bar{y})$ for every proper partition $\bar{x}^{\wedge} \bar{y}$ of $\bar{z}$. We let $\mathcal{M} A(M)$ denote the set of all mutually algebraic $L(M)$-formulas. When $M$ is understood, we simply write $\mathcal{M} A$.

The reader is cautioned that whether a formula $\varphi(\bar{z})$ is mutually algebraic or not depends on the choice of free variables. In particular, mutual algebraicity is not preserved under adjunction of dummy variables. Note that every $L(M)$-formula $\varphi(z)$ with exactly one free variable symbol is mutually algebraic. Furthermore, note that inconsistent formulas are mutually algebraic.

The following lemma indicates some of the closure properties of the set $\mathcal{M} A$. In what follows, when we write $\varphi(\bar{x}, \bar{y}) \in \mathcal{M} A$, we mean that $\varphi(\bar{z}) \in \mathcal{M} A$ for any tuple $\bar{z}$ of distinct symbols such that $\operatorname{range}(\bar{z})=\operatorname{range}(\bar{x}) \cup \operatorname{range}(\bar{y})$, but that we are concentrating on a specific proper partition $\bar{z}=\bar{x}^{\wedge} \bar{y}$ of $\varphi(\bar{z})$.

Lemma 1.3 Let $M$ be any structure in any language $L$.

1. If $\varphi(\bar{z}) \in \mathcal{M} A$, then $\varphi(\sigma(\bar{z})) \in \mathcal{M} A$ for any permutation $\sigma$ of the variable symbols.

2. If $\varphi(\bar{x}, \bar{y}) \in \mathcal{M} A$ and $\bar{a} \in M^{\lg (\bar{y})}$, then both $\exists \bar{y} \varphi(\bar{x}, \bar{y})$ and $\varphi(\bar{x}, \bar{a}) \in \mathcal{M} A$.

3. If $\varphi(\bar{z}) \vdash \psi(\bar{z})$ and $\psi(\bar{z}) \in \mathcal{M} A$, then $\varphi(\bar{z}) \in \mathcal{M} A$.

4. For $k \geq 1$, if $\left\{\varphi_{i}\left(\bar{z}_{i}\right): i<k\right\} \subseteq \mathcal{M} A$, and $\bigcap_{i<k}$ range $\left(\bar{z}_{i}\right)$ is nonempty, then $\psi(\bar{w}):=\bigwedge_{i<k} \varphi_{i}\left(\bar{z}_{i}\right) \in \mathcal{M} A$, where range $(\bar{w})=\bigcup_{i<k} \operatorname{range}\left(\bar{z}_{i}\right)$.

5. If $\varphi(\bar{x}, \bar{y}) \in \mathcal{M} A$ and $r \in \omega$, then $\theta_{r}(\bar{y}):=\exists \geq r \bar{x} \varphi(\bar{x}, \bar{y}) \in \mathcal{M} A$.

Definition 1.4 Given an arbitrary $L$-structure $M$, let $\mathcal{M} A^{*}(M)$ denote the set of all $L(M)$-formulas that are $T(M)$-equivalent to a Boolean combination of formulas from $\mathcal{M} A(M)$. A structure $M$ is mutually algebraic if $L(M)=\mathcal{M} A^{*}(M)$; that is, every $L(M)$-formula is $T(M)$-equivalent to a Boolean combination of mutually algebraic formulas.

It is evident that the mutual algebraicity of a structure is preserved under elementary equivalence. The following is the main theorem (Theorem 3.3) of [5].

Theorem 1.5 The following are equivalent for any theory $T$ :

1. Every model of $T$ is a mutually algebraic structure.

2. Every mutually algebraic expansion of every model of $T$ is a mutually algebraic structure.

3. $T h((M, A))$ has the non-finite cover property for every $M \models T$ and every expansion $(M, A)$ by a unary predicate.

4. Every complete extension of $T$ is weakly minimal and trivial. 
Next, we recall four classes of $L(M)$-formulas that were introduced in [4].

Definition 1.6 Let $M$ be any $L$-structure. We have

- $\mathcal{A}=$ all quantifier-free, mutually algebraic $L(M)$-formulas $\}$;

- $\mathcal{E}=\{$ all $L(M)$-formulas of the form $\exists \bar{x} \theta(\bar{x}, \bar{y})$, where $\theta \in \mathcal{A}\}$ (we allow $\lg (\bar{x})=0$ so $\mathcal{A} \subseteq \mathcal{E})$;

- $\mathcal{A}^{*}=\{$ all $L(M)$-formulas $T(M)$-equivalent to a Boolean combination of formulas from $\mathcal{A}\}$; and

- $\mathcal{E}^{*}=\{$ all $L(M)$-formulas $T(M)$-equivalent to a Boolean combination of formulas from $\mathcal{E}\}$.

The following theorem lists the main results of [4], specifically Proposition 4.1 and Theorem 4.2 (noting that by Theorem 1.5 , if $M$ is mutually algebraic, then $T h(M)$ is weakly minimal and trivial).

Theorem 1.7 Let $M$ be any mutually algebraic structure. Then we have the following:

1. Every quantifier-free $L(M)$-formula $\theta(\bar{z})$ is in $\mathcal{A}^{*}$.

2. Every $L(M)$-formula is $T(M)$-equivalent to a Boolean combination of formulas from $\mathcal{E}$, that is, $\mathcal{E}^{*}=L(M)$.

\section{A New Class of Existential Formulas}

We begin this section with the central definitions of the current note.

Definition 2.1 A formula $S(\bar{w})$ is a partial equality diagram if it is a Boolean combination of formulas of the form $w=w^{\prime}$ for various $w, w^{\prime} \in \bar{w}$.

An $L(M)$-formula $\theta(\bar{y}, \bar{z})$ is preferred if it has the form

$$
\exists \bar{x}(R(\bar{x}, \bar{y}) \wedge S(\bar{x}, \bar{y}, \bar{z})),
$$

where $\bar{x}, \bar{y}, \bar{z}$ are disjoint tuples of variable symbols, $\lg (\bar{y}) \geq 1, R(\bar{x}, \bar{y}) \in \mathcal{A}$, and $S(\bar{x}, \bar{y}, \bar{z})$ is a partial equality diagram.

Let $\mathcal{P}$ denote the set of all $L(M)$-formulas that are $T(M)$-equivalent to a positive Boolean combination of preferred formulas.

As the quantification in a preferred formula is only over the mutually algebraic conjunct, it is easily checked that every $\varphi(\bar{y}) \in \mathcal{P}$ is $T(M)$-equivalent to an algebraically existential formula in the sense of the footnote.

Lemma 2.2 Suppose that $M$ is an infinite, mutually algebraic structure, $\bar{x}, \bar{z}, y$ are disjoint sequences of variable symbols, $\lg (y)=1$, and $\left\{R_{j}\left(\bar{x}_{j}, y, \bar{z}_{j}\right)\right.$ : $j \in J\}$ is a finite set of quantifier-free, mutually algebraic formulas where, for each $j, \bar{x}_{j} \subseteq \bar{x}, \bar{z}_{j} \subseteq \bar{z}$, and the variable $y$ occurs in $R_{j}$. Then $T(M) \models$ $\forall \bar{x} \forall y \exists \bar{z} \bigwedge_{j \in J} \neg R_{j}\left(\bar{x}_{j}, y, \bar{z}_{j}\right)$.

Proof Given such a set of formulas, choose $N \succeq M$ and $\bar{a}, b$ from $N$. We will produce a tuple $\bar{e}$ from $N$ so that $N \models \neg R\left(\bar{a}_{j}, b, \bar{e}_{j}\right)$ for each $j \in J$. Say that $\bar{z}=\left(z_{0}, \ldots, z_{k-1}\right)$. For each $\ell<k$, let $J_{\ell}=\left\{j \in J: z_{\ell}\right.$ occurs in $\left.\bar{z}_{j}\right\}$, and let

$$
B_{\ell}:=\left\{c \in N: N \models \exists \bar{z}_{j}\left[R_{j}\left(\bar{a}_{j}, b, \bar{z}_{j}\right) \wedge z_{\ell}=c\right] \text { for some } j \in J_{\ell}\right\} .
$$

As each $R_{j}$ is mutually algebraic and $b$ is fixed, it follows that each of the sets $B_{\ell}$ is finite. Since $N$ is infinite, we can choose $\bar{e}=\left(e_{0}, \ldots, e_{k-1}\right)$ so that $e_{\ell} \notin B_{\ell}$ for each $\ell<k$. It is easily checked that $\bar{e}$ is as desired. 
Lemma 2.3 Let $M$ be an infinite, mutually algebraic structure. Say that

$$
\psi(\bar{x}, y):=\bigwedge_{i \in I} R_{i}\left(\bar{x}_{i}, y\right) \wedge \bigwedge_{j \in J} \neg R_{j}\left(\bar{x}_{j}, y\right),
$$

where $I$ and $J$ are finite, each $R_{i}, R_{j}$ is quantifier-free and mutually algebraic, each $\bar{x}_{i}$ and $\bar{x}_{j}$ is a subsequence of $\bar{x}, \lg (y)=1$, and $y$ occurs in each $R_{i}, R_{j}$. Then $\exists \bar{x} \psi(\bar{x}, y) \in \mathcal{P}$.

Proof First, if $I=\emptyset$, then by Lemma 2.2, T(M) $\models \forall y \exists \bar{x} \psi(\bar{x}, y)$; hence $\exists \bar{x} \psi(\bar{x}, y)$ is true for every $y$. In this case, $\exists \bar{x} \psi$ is equivalent to $y=y$, which is in $\mathcal{A}$, and hence in $\mathcal{P}$.

Next, assume that $I \neq \emptyset$. Let $\bar{x}^{\prime}$ be the smallest subsequence of $\bar{x}$ for which every $\bar{x}_{i}$ is a subsequence of $\bar{x}^{\prime}$. Let $\bar{z}=\bar{x} \backslash \bar{x}^{\prime}$, let $K=\left\{j \in J: \bar{x}_{j} \subseteq \bar{x}^{\prime}\right\}$, and let $J^{*}=J \backslash K$. As $I$ is nonempty, it follows from Lemma 1.3(3) and (4) that the formula

$$
\theta\left(\bar{x}^{\prime}, y\right):=\bigwedge_{i \in I} R_{i}\left(\bar{x}_{i}, y\right) \wedge \bigwedge_{j \in K} \neg R_{j}\left(\bar{x}_{j}, y\right)
$$

is mutually algebraic (and it is visibly quantifier-free). But, by Lemma 2.2, it follows that $\exists \bar{x} \psi(\bar{x}, y)$ is $T(M)$-equivalent to $\exists \bar{x}^{\prime} \theta\left(\bar{x}^{\prime}, y\right)$, so $\exists \bar{x} \psi(\bar{x}, y) \in \mathcal{P}$.

Theorem 2.4 The following are equivalent for every mutually algebraic structure $M$ :

1. $\exists=r \bar{x} R(\bar{x}, y) \in \mathcal{P}$ for all $R(\bar{x}, y) \in \mathcal{A}$ with $\lg (y)=1$ and all $r \in \omega$;

2. $\exists^{=r} \bar{x} R(\bar{x}, y) \in \mathcal{P}$ for all $R(\bar{x}, y) \in \mathcal{A}$ with $\lg (y) \geq 1$ and all $r \in \omega$;

3. $\mathcal{P}$ is closed under negation;

4. $\mathcal{P}=L(M)$;

5. $T(M)$ is model complete.

Proof First, note that if the universe of $M$ is finite, then all five conditions hold trivially. Thus, we assume throughout that $M$ is infinite.

$(1) \Rightarrow(2)$ : Assume that (1) holds. Choose any $R(\bar{x}, \bar{y}) \in \mathcal{A}$ and any integer $r$. Choose any variable symbol $y^{*} \in \bar{y}$, and let $\bar{y}^{\prime}$ satisfy $\bar{y}^{\prime \wedge} y^{*}=\bar{y}$. Choose an integer $N$ so that $R\left(\bar{x} \bar{y}^{\prime}, y^{*}\right)$ has fewer than $N$ solutions. For each $m<N$, let

$$
\begin{aligned}
& S_{m}\left(\bar{u}_{0} \bar{v}_{0} \cdots \bar{u}_{m-1} \bar{v}_{m-1}, \bar{y}^{\prime}\right) \\
& :=\bigwedge_{i \neq j} \bar{u}_{i} \bar{v}_{i} \neq \bar{u}_{j} \bar{v}_{j} \wedge \bigvee_{Q \in\left(\begin{array}{c}
m \\
r
\end{array}\right)}\left(\bigwedge_{i \in Q} \bar{v}_{i}=\bar{y}^{\prime} \wedge \bigwedge_{i \notin Q} \bar{v}_{i} \neq \bar{y}^{\prime}\right),
\end{aligned}
$$

and let

$$
\theta_{m}(\bar{y}):=\exists \bar{u}_{0} \bar{v}_{0} \cdots \exists \bar{u}_{m-1} \bar{v}_{m-1}\left(\bigwedge_{i<m} R\left(\bar{u}_{i} \bar{v}_{i}, y^{*}\right) \wedge S_{m}\left(\bar{u}_{0} \bar{v}_{0} \cdots \bar{u}_{m-1} \bar{v}_{m-1} \bar{y}^{\prime}\right)\right) .
$$

Using the closure properties in Lemma $1.3, \theta_{m}(\bar{y})$ is a preferred formula. Let $\bar{w}$ be new variables satisfying $\lg (\bar{w})=\lg (\bar{u})+\lg (\bar{v})$, and let $\delta(\bar{y})$ be

$$
\bigvee_{m<N}\left(\exists^{=m} \bar{w} R\left(\bar{w}, y^{*}\right) \wedge \theta_{m}(\bar{y})\right)
$$

It is easily checked that $\delta(\bar{y})$ is $T(M)$-equivalent to $\exists^{=r} \bar{x} R(\bar{x}, \bar{y})$ and, using (1), $\delta(\bar{y}) \in \mathcal{P}$.

$(2) \Rightarrow(3)$ : In order to show that $\mathcal{P}$ is closed under negation, by De Morgan's laws it suffices to show that the negation of every preferred formula is in $\mathcal{P}$. So 
fix a preferred formula $\theta(\bar{y}, \bar{z}):=\exists \bar{x}(R(\bar{x}, \bar{y}) \wedge S(\bar{x}, \bar{y}, \bar{z}))$, where $R(\bar{x}, \bar{y}) \in \mathcal{A}$, $\lg (\bar{y}) \geq 1$, and $S(\bar{x}, \bar{y}, \bar{z})$ is a partial equality diagram. Choose $N$ so that $T(M)$ implies that $\exists^{<N} \bar{x} R(\bar{x}, \bar{y})$. It is easily checked that $\neg \theta(\bar{y}, \bar{z})$ is $T(M)$-equivalent to

$$
\bigvee_{m<N}\left(\exists^{=m} \bar{x} R(\bar{x}, \bar{y}) \wedge \psi_{m}(\bar{y}, \bar{z})\right),
$$

where

$$
\psi_{m}(\bar{y}, \bar{z}):=\exists \bar{x}_{0} \cdots \bar{x}_{m-1}\left(\bigwedge_{i<m} R\left(\bar{x}_{i}, \bar{y}\right) \wedge \bigwedge_{i \neq j} \bar{y}_{i} \neq \bar{y}_{j} \wedge \bigwedge_{i<m} \neg S\left(\bar{x}_{i}, \bar{y}, \bar{z}\right)\right) .
$$

Thus, $\neg \theta(\bar{y}, \bar{z}) \in \mathscr{P}$ by (2).

(3) $\Rightarrow$ (4): As $\mathcal{P}$ is closed under positive Boolean combinations by definition, it follows immediately from (3) that $\mathcal{P}$ is closed under all Boolean combinations. However, $\mathcal{E} \subseteq \mathcal{P}$ trivially, so $\mathcal{E}^{*}$, the closure of $\mathcal{E}$ under Boolean combinations, is also a subset of $\mathcal{P}$. But, as $M$ is mutually algebraic, $\mathcal{E}^{*}=L(M)$ by Theorem 1.7(2). Thus $\mathcal{P}=L(M)$.

(4) $\Rightarrow(5)$ : Visibly, every preferred formula is an existential $L(M)$-formula, and the set of existential $L(M)$-formulas is closed under positive Boolean combinations. Thus, (4) implies that every $L(M)$-formula is $T(M)$-equivalent to an existential formula, which is equivalent to model completeness (see, e.g., Chang and Keisler [1]).

(5) $\Rightarrow$ (1): Assume that $T(M)$ is model complete. We argue that every $L(M)$-formula $\varphi(y)$ with $\lg (y)=1$ is in $\mathcal{P}$. Fix such a formula $\varphi(y)$.

Claim For any $N \succeq M$ and any $b \in N$ such that $N \models \varphi(b)$, there is $\delta(y) \in \mathcal{P}$ such that $N \models \delta(b) \wedge \forall y(\delta(y) \rightarrow \varphi(y))$.

Proof Fix such an $N$ and $b$. As $T(M)$ is model complete, this implies that

$$
T(M) \cup \Delta_{M} * \models \varphi(b),
$$

where $\Delta_{M} *$ is the atomic diagram of $M^{*}$. Thus, by compactness, there is a quantifier-free $\theta(\bar{e}, b) \in \Delta_{M} *$ such that $T(M) \cup\{\theta(\bar{e}, b)\} \models \varphi(b)$. Without loss of generality, we may assume that $\bar{e}$ is disjoint from $M \cup\{b\}$, so it follows that $T(M) \models \forall y(\exists \bar{x} \theta(\bar{x}, y) \rightarrow \varphi(y))$.

By Theorem 1.7(1), $\theta(\bar{x}, y) \in \mathcal{A}^{*}$. Thus, by considering the disjunctive normal form, we can write $\theta(\bar{x}, y)$ as $\bigvee \wedge R_{i j}\left(\bar{z}_{i j}\right)$, where each $\bar{z}_{i j}$ is contained in $\bar{x} \cup\{y\}$, and where $R_{i j}\left(\bar{z}_{i j}\right)$ is quantifier-free and is either mutually algebraic or is the negation of a mutually algebraic formula.

Thus, one of the disjuncts $\psi\left(\bar{x}^{\prime}, y\right)$ of $\theta(\bar{x}, y)$ satisfies $N \models \exists \bar{x}^{\prime} \psi\left(\bar{x}^{\prime}, b\right)$,

$$
T(M) \models \forall y\left(\exists \bar{x}^{\prime} \psi\left(\bar{x}^{\prime}, y\right) \rightarrow \varphi(y)\right),
$$

and $\bar{x}^{\prime} \subseteq \bar{x}$. Now $\psi\left(\bar{x}^{\prime}, y\right)$ has the form

$$
\bigwedge R_{i}\left(\bar{x}_{i}, y\right) \wedge \bigwedge \neg R_{j}\left(\bar{x}_{j}, y\right),
$$

where each $R_{i}$ and $R_{j}$ is quantifier-free and mutually algebraic, and each $\bar{x}_{i}, \bar{x}_{j} \subseteq$ $\bar{x}^{\prime}$. We may additionally assume that the variable symbol $y$ appears in each $R_{i}$ and $R_{j}$. As $M$ is infinite, Lemma 2.3 applies, and the formula $\delta(y):=\exists \bar{x}^{\prime} \psi\left(\bar{x}^{\prime}, y\right) \in \mathcal{P}$ is as required.

To finish the proof of (5) $\Rightarrow(1)$, let

$$
\Gamma:=\{\delta(y) \in \mathcal{P}: T(M) \models \forall y(\delta(y) \rightarrow \varphi(y))\} .
$$


It follows immediately from the Claim and compactness that the formula $\varphi(y)$ is $T(M)$-equivalent to a finite disjunction $\bigvee_{i} \delta_{i}(y)$ of elements $\delta_{i} \in \Gamma$. As $\mathcal{P}$ is closed under $T(M)$-equivalence and finite disjunctions, we conclude that $\varphi(y) \in \mathcal{P}$.

\section{New Proofs of Model Completeness}

We close by giving new proofs of the model completeness results first proved in [3] and [2]. The first theorem clearly follows from the second, but we give a separate proof as it follows so easily from our main result.

Theorem 3.1 If $T$ is strongly minimal and trivial, then $T(M)$ is model complete and $L(M)=\mathcal{P}$ for every model $M$ of $T$.

Proof Fix a model $M$ of $T$. With our eye on clause (1) of Theorem 2.4, choose an $L(M)$-formula $\varphi(y)$ with $\lg (y)=1$. By strong minimality, the solution set $\varphi(N)$ in any $N \succeq M$ is either finite or cofinite, with the "exceptional set" contained in $M$. That is, there is some finite set $Q \subseteq M$ such that, letting $\theta(y):=\bigvee_{m \in Q} y=m, \varphi(y)$ is $T(M)$-equivalent to either $\theta(y)$ or $\neg \theta(y)$. As any quantifier-free $L(M)$-formula in a single free variable is in $\mathcal{A}$ and hence in $\mathcal{P}$, both $\theta, \neg \theta \in \mathcal{P}$. Applying this argument to any instance of $\exists^{=r} \bar{z} R(\bar{z}, y)$, we conclude that both $T(M)$ is model complete and $L(M)=\mathscr{P}$ by Theorem 2.4.

Theorem 3.2 Suppose that $T$ is $\boldsymbol{\aleph}_{1}$-categorical, trivial, and of Morley rank 1 . Then for every $M \models T$, the elementary diagram is model complete. Furthermore, $L(M)=\mathcal{P}$.

Proof Again, we employ Theorem 2.4, but here we need to focus on a particular instance of clause (1). So fix a formula $R(\bar{z}, y) \in \mathcal{A}$ and an integer $r$. As $\exists \geq r \bar{z} R(\bar{z}, y) \in \mathcal{P}$, to establish clause (1) it suffices to prove that $\exists \leq r \bar{z} R(\bar{z}, y) \in \mathcal{P}$.

Toward this end, our assumptions on $T$ imply that there are finitely many nonalgebraic 1-types over $M$. Indeed, if $S_{\mathrm{na}}:=\left\{p_{i}: i<d\right\}$ denotes this set of nonalgebraic 1-types, then $d$ is the Morley degree of $T$. As well, the $\aleph_{1}$-categoricity of $T$ implies that each of these types are nonorthogonal. As $T$ is trivial, this further implies that $p_{i}$ and $p_{j}$ are not weakly orthogonal over $M$ for all $p_{i}, p_{j} \in S_{\text {na. }}$. As forking of a 1-type implies algebraicity, this implies that for any $N \succeq M$ and any $a \in p_{i}(N)$, there is $b \in p_{j}(N)$ such that $b \in \operatorname{acl}(M \cup\{a\})$ (and hence $a \in \operatorname{acl}(M \cup\{b\})$ ). As $\varepsilon^{*}=L(M)$, it is easy to verify that for all pairs $p_{i}, p_{j} \in S_{\text {na }}$, there is a mutually algebraic, quantifier-free formula $\theta_{i j}(x, y, \bar{z})$ such that for any $a \in p_{i}(N)$, there is $b \in p_{j}(N)$ such that $N \models \exists \bar{z} \theta_{i j}(a, b, \bar{z})$. Fix a finite set $\mathcal{F} \subseteq \mathcal{A}$ consisting of one such $\theta_{i j}$ for each pair $p_{i}, p_{j} \in S_{\text {na }}$ (if $i=j$, we can take $\theta_{i j}$ to be the mutually algebraic formula $x=y$ ).

Now, fix an elementary extension $N \succeq M$. For every $b \in N$, let

- $\theta^{*}(b, N)=\{c \in N: N \models \exists \bar{w} \theta(b, c, \bar{w})\}$, that is, $\theta^{*}(b, N)$ is the set of elements that are part of a tuple realizing $\theta(b, N)$;

- $\mathcal{F}(b)=\bigcup_{\theta \in \mathcal{F}} \theta^{*}(b, N)$; and

- $\mathcal{F}_{r}(b)=\left\{c \in \mathcal{F}(b): N \models \exists^{\geq r+1} \bar{z} R(\bar{z}, c)\right\}$.

Clearly, for $b \in N \backslash M, \mathcal{F}_{r}(b) \subseteq \mathcal{F}(b)$ and $|\mathcal{F}(b)| \leq \sum_{\theta \in \mathcal{F}} N_{\theta} \cdot \lg (\bar{z})$, where $N_{\theta}$ is an integer such that $T(M) \models \forall y \exists^{<N_{\theta}} \bar{z} \theta(y, \bar{z})$.

Thus, there is a finite exceptional set $Q \subseteq M$ and an integer $\ell^{*}$ such that

1. $T(M) \models \forall y\left(y \notin Q \rightarrow\left|\mathcal{F}_{r}(y)\right| \leq \ell^{*}\right)$, and 
2. for some $b \in N \backslash M,\left|\mathcal{F}_{r}(b)\right|=\ell^{*}$.

Also, it is clear that the size $\left|\mathcal{F}_{r}(b)\right|$ depends only on $\operatorname{tp}(b / M)$; that is, if $\operatorname{tp}(b / M)=\operatorname{tp}\left(b^{\prime} / M\right)$, then $\left|\widetilde{F}_{r}(b)\right|=\left|\mathcal{F}_{r}\left(b^{\prime}\right)\right|$. Fix any nonalgebraic 1-type $p^{*}(y) \in S_{\text {na }}$ such that $\left|\mathscr{F}_{r}(b)\right|=\ell^{*}$ for some (every) realization $b$ of $p^{*}$.

Let $\delta(x)$ express

"There is some $\theta(x, y, \bar{z}) \in \mathcal{F}$ such that $\exists y \exists \bar{z}(\theta(x, y, \bar{z}) \wedge y \notin Q$ and there are distinct elements $\left\{w_{i}: i<\ell^{*}\right\}$ witnessing that $\left|\mathscr{F}_{r}(y)\right| \geq \ell^{*}$ and $x \neq w_{i}$ for all $\left.i<\ell^{*}\right)$."

It is routine to check that the formula $\delta(x) \in \mathcal{P}$. It suffices to prove the following.

Claim $\quad T(M) \models \forall x[\exists \leq r \bar{z} R(\bar{z}, x) \leftrightarrow \delta(x)]$.

Proof Fix any $N \succeq M$ and $a \in N$. First, suppose that $N \models \exists \leq r \bar{z} R(\bar{z}, a)$. Choose $\theta(x, y, \bar{z}) \in \mathscr{F}$ such that there is $b \in p^{*}(N)$ with $N \models \exists \bar{z} \theta(a, b, \bar{z})$. By our choice of $p^{*}$ we have $\left|\mathcal{F}_{r}(b)\right|=\ell^{*}$, so choose an enumeration $\left\{c_{i}: i<\ell^{*}\right\}$ of $\mathcal{F}_{r}(b)$. Since the definition of $\mathscr{F}_{r}(b)$ implies that $N \models \exists \geq r+1 \bar{z} R\left(\bar{z}, c_{i}\right)$ for each $i$, it follows that $a \neq c_{i}$ for each $i$. Thus, $N \models \delta(a)$.

Conversely, suppose that $N \models \exists^{\geq r+1} \bar{z} R(\bar{z}, a)$. Choose any $b \in N \backslash Q$ such that $N \models \exists \bar{z} \theta(a, b, \bar{z})$ for some $\theta \in \mathcal{F}$ and $\left|\mathcal{F}_{r}(b)\right| \geq \ell^{*}$. Choose any set of $\ell^{*}$ distinct elements $\left\{c_{i}: i<\ell^{*}\right\} \subseteq \mathcal{F}_{r}(b)$. But now, as $b \notin Q$,

$$
N \models\left|\mathcal{F}_{r}(b)\right| \leq \ell^{*} .
$$

This, combined with the fact that our assumption on $a$ and $\theta$ implies that $a \in \mathcal{F}_{r}(b)$, guarantees that $a=c_{i}$ for some $i$. That is, $N \models \neg \delta(a)$, completing the proof of the Claim.

As we have shown that $\exists \leq r \bar{z} R(\bar{z}, x) \in \mathcal{P}$, it follows from Theorem 2.4 that both $T(M)$ is model complete and $L(M)=\mathscr{P}$.

\section{Note}

1. Every $\varphi(\bar{y}) \in \mathcal{P}$ can be written in the form $\exists \bar{x} \psi(\bar{x}, \bar{y})$, where $\psi$ is quantifier-free and there is an integer $K$ so that $T(M) \models \forall \bar{y} \exists^{<K} \bar{x} \psi(\bar{x}, \bar{y})$. Perhaps such a formula should be called an "algebraically existential" formula?

\section{References}

[1] Chang, C. C., and H. J. Keisler, Model Theory, North Holland, Amsterdam, 1973. 467

[2] Dolich, A., M. C. Laskowski, and A. Raichev, "Model completeness for trivial, uncountably categorical theories of Morley rank 1," Archive for Mathematical Logic, vol. 45 (2006), pp. 931-45. Zbl 1111.03035. MR 2271332. DOI 10.1007/s00153-006-0019-x. 463,468

[3] Goncharov, S. S., V. S. Harizanov, M. C. Laskowski, S. Lempp, and C. F. D. McCoy, "Trivial, strongly minimal theories are model complete after naming constants," Proceedings of the American Mathematical Society, vol. 131 (2003), pp. 3901-12. Zbl 1035.03013. MR 1999939. DOI 10.1090/S0002-9939-03-06951-X. 463, 468

[4] Laskowski, M. C., "The elementary diagram of a trivial, weakly minimal structure is near model complete," Archive for Mathematical Logic, vol. 48 (2009), pp. 15-24. Zbl 1162.03018. MR 2480932. DOI 10.1007/s00153-008-0116-0. 463, 464, 465

[5] Laskowski, M. C., "Mutually algebraic structures and expansions by predicates," Journal of Symbolic Logic, vol. 78 (2013), pp. 185-94. Zbl 1261.03119. MR 3087069. DOI 10.2178/jsl.7801120. 463, 464 


\section{Acknowledgment}

The author was partially supported by National Science Foundation grant DMS-0901336.

Department of Mathematics

University of Maryland

College Park, Maryland 20742

USA

mcl@math.umd.edu 